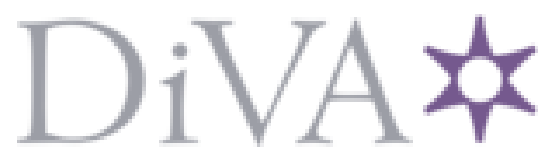

http://www.diva-portal.org

This is the published version of a paper published in Physical Review A. Atomic, Molecular, and Optical Physics.

Citation for the original published paper (version of record):

Andersson, E., Fritzsche, S., Linusson, P., Hedin, L., Eland, J H. et al. (2010)

Multielectron coincidence study of the double Auger decay of 3d-ionized krypton.

Physical Review A. Atomic, Molecular, and Optical Physics, 82(4): 043418

http://dx.doi.org/10.1103/PhysRevA.82.043418

Access to the published version may require subscription.

N.B. When citing this work, cite the original published paper.

Permanent link to this version:

http://urn.kb.se/resolve?urn=urn:nbn:se:uu:diva- 122568 


\title{
Multielectron coincidence study of the double Auger decay of $3 d$-ionized krypton
}

\author{
E. Andersson, ${ }^{1}$ S. Fritzsche, ${ }^{2,3}$ P. Linusson, ${ }^{4}$ L. Hedin, ${ }^{1}$ J. H. D. Eland, ${ }^{5}$ J.-E. Rubensson, ${ }^{1}$ L. Karlsson, ${ }^{1}$ and R. Feifel ${ }^{1}$ \\ ${ }^{1}$ Department of Physics and Astronomy, Uppsala University, Box 516, SE-751 20 Uppsala, Sweden \\ ${ }^{2}$ Department of Physics, P.O. Box 3000, Fin-90014 University of Oulu, Finland \\ ${ }^{3}$ GSI Helmholtzzentrum für Schwerionenforschung, D-64291 Darmstadt, Germany \\ ${ }^{4}$ Department of Physics, Stockholm University, AlbaNova University Centre, SE-106 91 Stockholm, Sweden \\ ${ }^{5}$ Department of Chemistry, Physical and Theoretical Chemistry Laboratory, Oxford University, South Parks Road, \\ Oxford OX1 3QZ, United Kingdom
}

(Received 7 April 2010; published 19 October 2010)

\begin{abstract}
Multielectron coincidence data for triple ionization of krypton have been recorded above the $3 d$ ionization threshold at two photon energies (140 and $150 \mathrm{eV}$ ). Three principal transition pathways have been observed, two involving double Auger transitions from $\mathrm{Kr}^{+}$, and one involving single Auger transitions from $\mathrm{Kr}^{2+}$ created by direct single-photon double ionization. The decay of the $3 d^{9}{ }^{2} D_{5 / 2,3 / 2}$ states in $\mathrm{Kr}^{+}$has been analyzed in some detail and is found to be strongly dominated by cascade processes where two electrons with well-defined energies are emitted. The decay paths leading to the $4 s^{2} 4 p^{3}{ }^{4} S,{ }^{2} D$, and ${ }^{2} P$ states of $\mathrm{Kr}^{3+}$ are analyzed and energies of seven intermediate states in $\mathrm{Kr}^{2+}$ are given. A preliminary investigation of the decay paths from $\mathrm{Kr}^{+} 3 d^{9} 4 p^{5} n l$ shake-up states has also been carried out.
\end{abstract}

DOI: 10.1103/PhysRevA.82.043418

PACS number(s): $32.80 . \mathrm{Fb}, 32.80 . \mathrm{Zb}, 34.50 . \mathrm{Gb}$

\section{INTRODUCTION}

Single-photon absorption by matter can result in the emission of one electron, as originally explained by Einstein in 1905 in his photoelectric law [1], or in the emission of several electrons as discovered by Auger in 1925 [2]. If one photoelectron and one Auger electron are emitted, the system ends up in a doubly charged state. Single-photon double ionization of this kind has been extensively studied by Auger spectroscopy (see, e.g., Ref. [3]). If two Auger electrons are emitted upon inner-shell photoionization, triply charged states, as first observed by Carlson and Krause in 1965 [4], can be created. Such double Auger emission occurs either simultaneously, which is then referred to as a direct double Auger process, or sequentially in the form of a cascade process. Generally, the formation of multiply charged systems is highly relevant to the behavior of matter in high-intensity radiation environments such as Earth's outer atmosphere and interstellar space.

In order to study the emitted photoelectron and the related Auger electrons simultaneously, one can make use of coincidence methods which give important information on the transition pathways. One of today's most powerful multielectron coincidence techniques is based on a magnetic bottle for the measurement of electron flight times [5], as recently introduced by Eland et al. [6] and implemented at synchrotron radiation sources for inner-shell studies by Penent et al. [7]. The magnetic bottle technique makes it possible to obtain detailed information on the photoionized states as well as various decay paths as has been demonstrated by Penent et al. [7] for the Xe $4 d$ case and by Lablanquie et al. for the $\operatorname{Ar} 2 p$ case [8]. A major strength of coincidence experiments is the possibility to project specific signals from the multidimensional data sets, where signals from competing processes may overlap with each other and hide features of interest, as we shall demonstrate in this work. In the work of Penent et al. [7], three strong resonances in the cascade Auger decays of $\mathrm{Xe}^{+} 4 d^{9}$ to $\mathrm{Xe}^{3+} 5 p^{3}$ were revealed. For the double
Auger decay of $3 d$ ionized $\mathrm{Kr}$, which is the case of interest in the present study, two previous studies $[9,10]$ indicate the existence of similar resonances as in the Xe case, but they provide no identification and no energies of the intermediate states involved. These intermediate state resonances involved in the cascade Auger decays of $\mathrm{Kr}^{+} 3 d^{9}$ will be investigated in detail in this work.

\section{EXPERIMENTAL DETAILS}

The experiments were performed at beamline U49/2 PGM 2 [11] at the BESSY-II storage ring, Germany, using essentially the same setup as has previously been described (see, e.g., Ref. [12] and references therein). Briefly, multielectron coincidences were detected using a magnetic bottle timeof-flight spectrometer [6] capable of resolving individual electron kinetic energies. The resolving power of the apparatus for single electrons can be expressed as a fixed numerical resolution $E / \Delta E$ of about 50 for electron energies above $1 \mathrm{eV}$, and a fixed resolution $\Delta E$ of about $20 \mathrm{meV}$ at lower energies. Commercially obtained krypton gas was let into the interaction region of the spectrometer through a hypodermic needle and then ionized by 140 and $150 \mathrm{eV}$ photons, respectively. The electron flight times were referenced to the synchrotron light pulses, which have a width of about $30 \mathrm{ps}$ and a periodicity of $800.5 \mathrm{~ns}$ [13] in single-bunch operation of the BESSY-II storage ring. The latter implies a repetition rate of the light source of about 1.25 MHz. In order to avoid accidental coincidences, the electron count rates were restricted to a few $\mathrm{kHz}$ (i.e., a small fraction of the ionizing light pulse rate). This was achieved by closing the exit slit of the monochromator, whereby the intensity of the synchrotron light was suitably reduced. As a consequence, the energy resolution of the light was about $20 \mathrm{meV}$ and hence not a limiting factor for the measurements. The monochromator was calibrated using literature values of the $\mathrm{Kr} 3 d$ near-edge x-ray absorption spectrum [14] while the time-to-energy conversion 
was calibrated using known energies of $\mathrm{Xe}$ and $\mathrm{Kr}$ Auger lines [15].

\section{THEORETICAL DETAILS}

To calculate the level structure of multiply charged ions, and, especially of hole states, the multiconfiguration DiracFock (MCDF) method has been found to be a versatile tool for providing good-to-reliable theoretical predictions on the excitation energies, Auger rates, and lifetimes. For medium and heavy elements, this method enables one to account for at least the dominant relativistic and many-body effects on an equal basis, including the rearrangement of the bound-state electron density if the atom undergoes some decay (cascade). In the MCDF method [16], an atomic state is approximated by a linear combination of configuration state functions (CSF) of the same total angular momentum $(J M)$ and parity $(P)$,

$$
\psi_{\alpha}(P J M)=\sum_{r=1}^{n_{c}} c_{r}(\alpha)\left|\gamma_{r} P J M\right\rangle,
$$

where $n_{c}$ is the number of CSFs and $\left\{c_{r}(\alpha)\right\}$ denotes the representation of the atomic state in the basis chosen. In most standard computations, the CSFs $\left|\gamma_{r} P J M\right\rangle$ are constructed as antisymmetrized products of a common set of orthonormal orbitals and are optimized together on the basis of the DiracCoulomb Hamiltonian. Relativistic effects due to the Breit interaction are then added to the representation $\left\{c_{r}(\alpha)\right\}$ by diagonalizing the Dirac-Coulomb-Breit Hamiltonian matrix $[17,18]$. Further corrections due to the self-energy and vacuum polarization of the electron density have been estimated but were found negligible compared to missing correlation contributions for the $3 d$ hole of krypton and its subsequent decay states. Apart from a rather restricted set of bound-state CSF, shifts in the relative energy of the various hole states arise also from their coupling to the continuum (of the next higher charge state, so-called "interchannel interactions") that is not taken into account in the standard MCDF procedure [19].

For ions with several inner or subvalence holes, the main limitations in applying the MCDF model typically arise from the rapidly increasing size of the wave-function expansion and the neglected coupling to the continuum. Indeed, the expansion [cf. Eq. (1)] often needs to be truncated quite seriously if a large number of low-lying levels are considered or if one searches for levels in the vicinity of an ionization limit. The size of the wave-function expansion also affects our ability to calculate Auger rates in order to estimate the population of intermediate states in the Auger cascade. In the present analysis, the structure code GRASP92 [17] has been employed to generate the wave functions for the levels of singly, doubly, and triply charged krypton, while the RATIP program [20] was utilized to obtain all the transition amplitudes and Auger rates. Starting from the reference configurations of a given ionic state, for example $4 s^{2} 4 p^{4}+4 s 4 p^{5}+4 s^{0} 4 p^{6}$ for doubly charged krypton, additional single $(S)$ and double $(D)$ excitations were incorporated into the expansion [cf. Eq. (1)] to capture further correlations. Unfortunately, however, a systematic incorporation of all $S D$ excitations into, for example, the next two layers of $(n l j)$ correlation orbitals is not possible at present. In order to identify some of the intermediate levels (see Fig. 7 below), a transformation of the wave functions into a $L S J$-coupled basis has been performed in addition to some of the levels [21].

A special effort has been made to identify the states of doubly charged krypton above the $4 p^{3}$ ionization limit of the next higher charge state, for example, the levels which decay further by Auger emission (see Fig. 7 and Sec. IV below). For these $4 l 4 l^{\prime}$ hole states and their low-lying excitations, it turns out that single excitations of the $4 s^{0} 4 p^{6}{ }^{1} \mathrm{~S}_{0}$ level are embedded into the continuum, while single excitations from the $4 s^{2} 4 p^{4}$ ground and $4 s 4 p^{5}$ excited configurations are below the ionization limit. Therefore, it is mainly the correlation states of the $4 p^{6}{ }^{1} S_{0}$ level that are expected to undergo Auger decay.

To explore the coarse-grained structure of the $\mathrm{Kr}^{2+}$ ions after the first Auger electron emission, two series of computations have been carried out, one for the single and a few double excitations of the nine levels from the $4 s^{2} 4 p^{4}+$ $4 s 4 p^{5}$ configurations, and a second computation for the correlation satellites of the $4 s^{0} p^{6}{ }^{1} S_{0}$ level (cf. Fig. 7 below). Apart from the level energies, we also calculated the Auger rates for the two steps of the cascade $3 d^{-12} D_{3 / 2,5 / 2} \longrightarrow$ $4 p^{6}{ }^{1} S_{0}(+\mathrm{SD}$ excitations $) \longrightarrow 4 s^{2} 4 p^{3}{ }^{4} S,{ }^{2} D,{ }^{2} P$. To obtain all the required Auger amplitudes, the AUGER component of the RATIP program was utilized in which the continuum orbitals for the emitted electrons are solved within a spherical but level-dependent potential of the final ion; see Refs. [22,23] for further details.

\section{RESULTS AND DISCUSSION}

A map of three-electron coincidence events, recorded at $150 \mathrm{eV}$ photon energy, is shown in Fig. 1 with the total kinetic energy sum $\left(\varepsilon_{1}+\varepsilon_{2}+\varepsilon_{3}\right)$ on the horizontal $(x)$ axis and the sum of the kinetic energies of the two slower electrons $\left(\varepsilon_{2}+\varepsilon_{3}\right)$ on the vertical $(y)$ axis. The map shows essentially nine groups of features arranged in a regular pattern. We shall refer to these features as spots. In the upper panel of the figure, the projection onto the $x$ axis is shown, which reveals three peaks reflecting the ${ }^{4} S_{3 / 2},{ }^{2} D_{5 / 2,3 / 2}$, and ${ }^{2} P_{3 / 2,1 / 2}$ final states of $\mathrm{Kr}^{3+}$ formed out of the $4 s^{2} 4 p^{3}$ configuration. Most of the line intensities originate from the six strong spots in the lower part of the coincidence map. These spots have a length of about $1.7 \mathrm{eV}$ in the $x$ direction due to the comparatively low resolution of the fastest electron, which blurs the spin-orbit fine structure. The width in the $y$ direction is only about $0.5 \mathrm{eV}$ since the uncertainty in $\varepsilon_{2}$ and $\varepsilon_{3}$ is much smaller than in $\varepsilon_{1}$ due to the low kinetic energy of these electrons. It is noteworthy that the kinetic energy of the fastest electron is lower further up in the map, and hence the spots are shorter there.

In the left panel, the $y$ projection shows three groups of lines labeled I, II, and III, respectively. They correspond to three distinct transition pathways to the electronic states associated with the $4 s^{2} 4 p^{3}$ configuration of $\mathrm{Kr}^{3+}$ as will be shown below.

Three basic mechanisms leading to triple ionization (release of three electrons) by the absorption of a single photon can be readily envisioned. They are (using the symbol * to indicate a highly excited system susceptible to Auger electron decay) as 


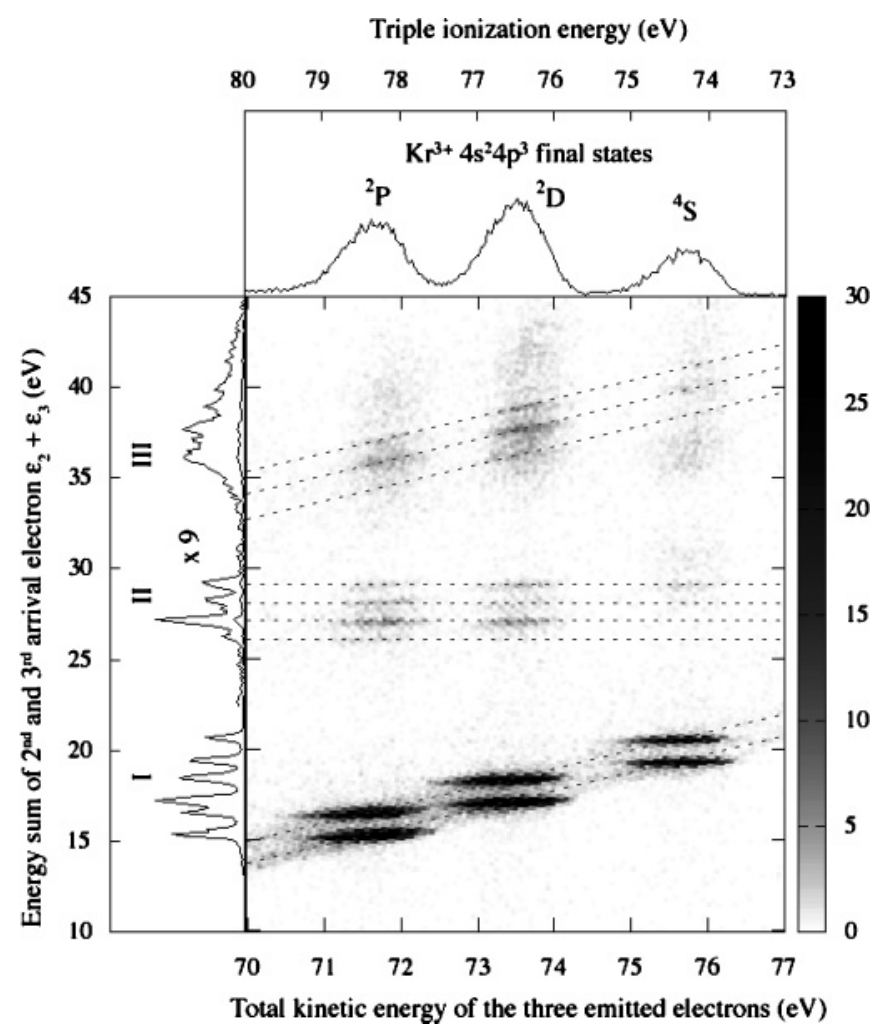

FIG. 1. Coincidence map revealing three distinct decay paths to the $4 s^{2} 4 p^{3}$ configuration of $\mathrm{Kr}^{3+}$ measured at $h v=150 \mathrm{eV}$. The $x$ axis represents the total kinetic energy sum of all three electrons detected, and a projection of the total data set onto this axis is shown in the upper panel. This projection reflects the $4 s^{2} 4 p^{3}{ }^{4} S,{ }^{2} D$, and ${ }^{2} P$ final states of $\mathrm{Kr}^{3+}$. The $y$ axis represents the kinetic energy sum of the two slower electrons, and the projection onto it, which is displayed in the left panel, shows three groups of lines (I, II, and III) corresponding to different decay paths as discussed in the text. In order to highlight the interrelation of the second and third arrival electrons, dashed lines have been included as guides for the eyes.

follows:

(1) Direct triple ionization:

$$
h v+A \rightarrow A^{3+}+3 e^{-} .
$$

(2) Direct double ionization with additional single Auger decay:

$$
h v+A \rightarrow\left(A^{2+}\right)^{*}+2 e^{-} ;\left(A^{2+}\right)^{*} \rightarrow A^{3+}+e^{-} .
$$

(3) Single inner-shell ionization with ensuing double Auger decay (direct or sequential):

$$
h v+A \rightarrow\left(A^{+}\right)^{*}+e^{-} ;\left(A^{+}\right)^{*} \rightarrow A^{3+}+2 e^{-} .
$$

The first of these is relatively simple. It has been studied for valence shells by Eland et al. [24], but it is found to be of minor significance in the present study where a core hole is involved. The transition paths associated with the two latter processes involving a $3 d$ inner shell hole are more complex. They can be subdivided into a primary photoionization process, possibly incorporating shake-ups, and secondary Auger processes, as shown schematically in Fig. 2.

In what follows we will mainly focus on triple ionization connected to single $3 d$ ionization with ensuing double Auger decay leading to three vacancies in the $4 p$ shell (mechanism 3 ). Such processes are represented by the lowest group of features in Fig. 1, group I, where the kinetic energy sum $\varepsilon_{2}+\varepsilon_{3}$ lies between 15 and $21 \mathrm{eV}$. The map shows three pairs of spots with a common separation of $1.2 \mathrm{eV}$. The kinetic energy measured for the fastest electron, $\varepsilon_{1}$, is $56.2 \mathrm{eV}$ for the lower spots and $55 \mathrm{eV}$ for the higher ones. These electrons are identified as the $3 d$ photoelectrons from single photoionization and the splitting of $1.2 \mathrm{eV}$ is due to the spin-orbit coupling of the $3 d$ single hole state.

The ionization energy of the $\mathrm{Kr}^{3+}$ final states is obtained as the photon energy minus the total kinetic energy of the three electrons emitted irrespective of transition pathway. These energies can be determined from the ionization energy scale in the upper panel of Fig. 1 and it should be noted that the energies (74.3, 76.5, and $78.3 \mathrm{eV}$ for ${ }^{4} S,{ }^{2} D$, and ${ }^{2} P$, respectively) are approximately $1 \mathrm{eV}$ below the corresponding values in NIST [25]. This discrepancy has been noted before [9,10,24] and our values agree very well with these previous works, if the rather large uncertainty in $\varepsilon_{1}$ is taken into account.

The comparatively large cross section of single ionization gives rise to false coincidences which show up in the lower part of the map as two weak and slanted bands over the whole total kinetic energy range plotted in Fig. 1. However, the intensity of the true triple coincidence events is greatly enhanced and forms the six strong spots discussed.

We next consider group II of the $y$ projection in Fig. 1 which shows four lines, where $\varepsilon_{2}+\varepsilon_{3}$ has values between 25 and $30 \mathrm{eV}$. It is apparent from the coincidence map that the kinetic energy sum of the second and third arrival electrons is independent of the kinetic energy of the first arrival electron, as marked by the dashed horizontal lines in Fig. 1. This suggests a two-step triple ionization process either in the form of mechanism 2 or as the direct double Auger of mechanism 3. However, the spectrum in the $y$ projection moves when the photon energy is changed, which suggests a direct double photoionization process where the two primary photoelectrons acquire lower energies than the electron released in the secondary Auger process. In support of this interpretation, the kinetic energy sum $\varepsilon_{2}+\varepsilon_{3}$ agrees well with the energies of the $3 d^{9} 4 p^{5}$ core-valence states of doubly ionized krypton, as previously observed by Bolognesi et al. [26]. This energy region will be discussed in detail in a separate paper [27].

The topmost group of features in the $y$ projection, group III, is a fairly wide distribution of lines observed between 29 and $45 \mathrm{eV}$. This energy range suggests that shake-up states related to the $3 d$ hole are involved. The main features resemble the lines of group I, which supports this interpretation, but the broad spread of intensity observed on the high-energy side of group III in Fig. 1 is at variance with the structure of group I. This increased complexity is related to the involvement of several intermediate shake-up electron configuration states. This will be further discussed in connection with Fig. 8 below. It may be noted that the corresponding lines in the coincidence map are slightly tilted. An explanation for the tilt can be found in the fact that the energies of the second and third arrival electrons are plotted on both the $x$ and $y$ axes. Because the kinetic energy sum of these electrons is high and that of the first electron is low, it has a significant spread due to the 


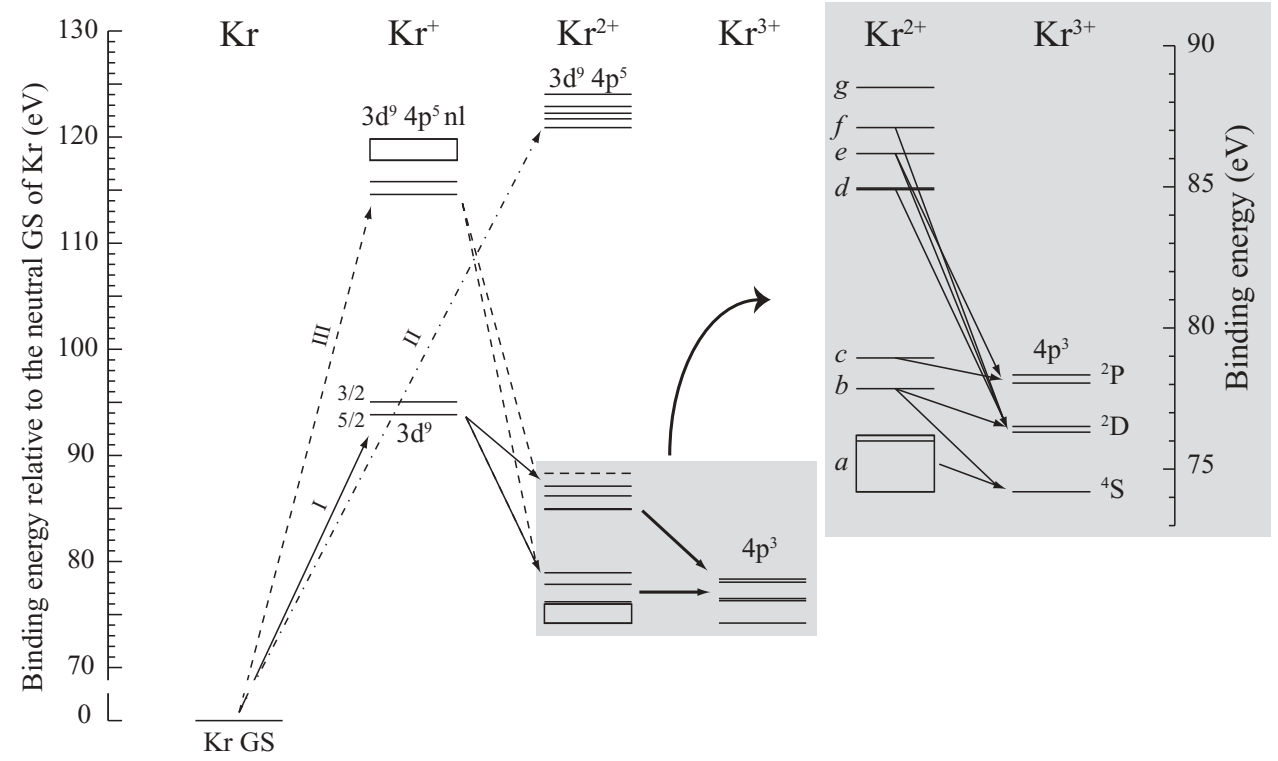

FIG. 2. Schematic energy level diagram of atomic krypton in various charge states. The arrows labeled I, II, and III show photoionization processes revealed in Fig. 1. Observed Auger cascade processes to $4 s^{2} 4 p^{3} \mathrm{Kr}^{3+}$ via $\mathrm{Kr}^{2+}$ intermediate states, labeled $a$ to $g$, are marked with arrows in the enlargement on the right-hand side.

instrumental resolution and produces spots of unit positive slope. By contrast in group I the instrumental energy spread of the first high-energy electron is dominant, producing nearly horizontal bands.

In Fig. 3 we focus on the double Auger decay of the $\mathrm{Kr}^{+}$ $3 d^{9}$ hole states with the kinetic energy of the slowest Auger electron, $\varepsilon_{3}$, plotted along the $x$ axis, and the sum of the kinetic energies of the two Auger electrons, $\varepsilon_{2}+\varepsilon_{3}$, plotted along the $y$ axis of this coincidence map. Two initial states $\left(3 d^{9}{ }^{2} D_{5 / 2,3 / 2}\right)$ and three final state terms $\left(4 s^{2} 4 p^{3}{ }^{4} S,{ }^{2} \mathrm{D}\right.$, and ${ }^{2} P$ ) are resolved, and hence there are essentially six horizontal bands in the coincidence map. The bands associated with ${ }^{2} D$ and ${ }^{2} P$ tricationic states show doublets due to spin-orbit interaction. The initial and final states associated with each band are readily identified using the energy differences listed in Table I.

The left panel in the figure shows the projection onto the $y$ axis of the map. It presents the line spectrum of group I

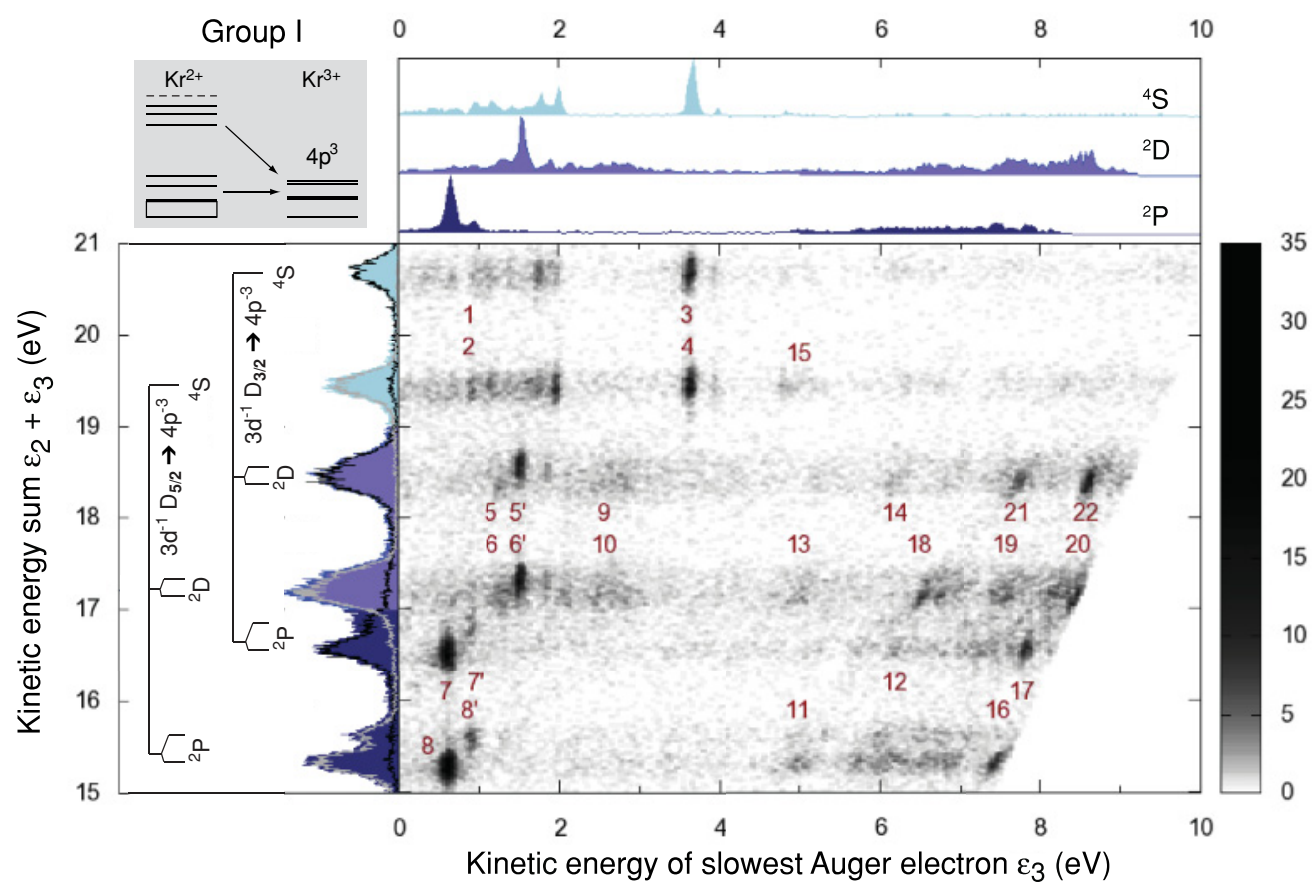

FIG. 3. (Color online) Triple ionization coincidence map of $\mathrm{Kr}$ selected on $3 d$ photoelectrons as the first arrival electron. The map shows the intensity as a function of the kinetic energy sum of the two Auger electrons (along $y$ ), and the kinetic energy of the slowest Auger electron (along $x$ ). The projection onto the $y$ axis is an enlargement of the line spectrum of group I from Fig. 1; color coding is used as explained in the text. The peaks corresponding to ${ }^{2} D$ and ${ }^{2} P$ show substructure due to spin-orbit interaction. The projections onto the $x$ axis, which are displayed in the upper panel, show the spectrum of the slowest electron in the decay to each of the three final tricationic state terms under consideration; the color coding is used consistently. Strong features in the map are numbered 1 to 22 and are listed in Table II. 
TABLE I. Measured energy differences between initial and final states (cf. left panel of Fig. 3). Energies are given with an uncertainty by \pm 1 within the first decimal.

\begin{tabular}{lcc}
\hline \hline $\begin{array}{l}\text { Initial state } \\
\mathrm{Kr}^{+} 3 d^{9}\end{array}$ & $\begin{array}{c}\text { Final state } \\
\mathrm{Kr}^{3+} 4 s^{2} 4 p^{3}\end{array}$ & $\begin{array}{c}\text { Energy difference } \\
(\mathrm{eV})\end{array}$ \\
\hline${ }^{2} D_{3 / 2}$ & ${ }^{4} S_{3 / 2}$ & 20.6 \\
${ }^{2} D_{5 / 2}$ & ${ }^{4} S_{3 / 2}$ & 19.4 \\
${ }^{2} D_{3 / 2}$ & ${ }^{2} D_{3 / 2}$ & 18.5 \\
${ }^{2} D_{3 / 2}$ & ${ }^{2} D_{5 / 2}$ & 18.3 \\
${ }^{2} D_{5 / 2}$ & ${ }^{2} D_{3 / 2}$ & 17.3 \\
${ }^{2} D_{5 / 2}$ & ${ }^{2} D_{5 / 2}$ & 17.1 \\
${ }^{2} D_{3 / 2}$ & ${ }^{2} P_{1 / 2}$ & 16.8 \\
${ }^{2} D_{3 / 2}$ & ${ }^{2} P_{3 / 2}$ & 16.5 \\
${ }^{2} D_{5 / 2}$ & ${ }^{2} P_{1 / 2}$ & 15.6 \\
${ }^{2} D_{5 / 2}$ & ${ }^{2} P_{3 / 2}$ & 15.3 \\
\hline \hline
\end{tabular}

from Fig. 1 in a zoomed version. A grayscale (color online) coding has been introduced to indicate the initial and final state associated with each band. Decays from the $3 d^{9}{ }^{2} D_{5 / 2}$ and ${ }^{2} D_{3 / 2}$ initial hole states of $\mathrm{Kr}^{+}$are plotted using a light and a dark line, respectively. The peaks corresponding to decays to $4 s^{2} 4 p^{3}{ }^{4} S$ are filled with a light color tone and the peaks corresponding to decays to ${ }^{2} D$ and ${ }^{2} P$ are filled with a medium and a dark color tone, respectively. The two peaks corresponding to the ${ }^{2} P$ tricationic final state are visibly split by spin-orbit interaction whereas for ${ }^{2} D$ the spin-orbit splitting gives rise to broadening. The upper panel shows the $x$ projection (i.e., the spectra of the slowest Auger electron of all final tricationic state terms under consideration using consistently the same color coding).

Strong features apparent in the map of Fig. 3 have been labeled by numbers, and the corresponding electron energies are listed in Table II. The dark spots in the map are distributed in an intricate pattern where some spots are aligned vertically and some lie along diagonal lines. The $x$ projection of each band in the coincidence map is plotted in Fig. 4 in more detail, where the kinetic energy of the slowest electron, $\varepsilon_{3}$, has been shifted by the binding energy of the various $4 s^{2} 4 p^{3}$ final state terms relative to the ${ }^{4} S$ tricationic ground state. The spectra of the associated faster Auger electron, $\varepsilon_{2}$, have been plotted in Fig. 5 and display structures corresponding to the ones in Fig. 4, bearing in mind that the energy resolution decreases with increasing electron kinetic energy.

In discussing these three figures in more detail, the following qualitative arguments are useful. If an atom decays by direct double Auger decay, the two emitted electrons can share the released energy arbitrarily, which gives rise to continuous structures in a coincidence map of the two electrons. In contrast, cascade Auger decays can be described as a two-step process where the electrons are emitted with discrete kinetic energies. A coincidence measurement of this process will reveal sharp structures, from which the energy level of the intermediate state in the decay can be deduced. It is not known a priori if the electron emitted in the first or in the second step has the highest kinetic energy, but the total available energy for both electrons emitted is defined by the energy difference between the initial and final states of a cascade (cf. Fig. 2 and Table I). As can be seen from Figs. 4 and 5, the spectra are dominated by discrete structures, which suggests that the $\mathrm{Kr} 3 d$ states decay primarily through cascade processes via intermediate discrete energy levels of $\mathrm{Kr}^{2+}$.

The topmost band of the coincidence map in Fig. 3, which corresponds to the topmost spectra in Figs. 4 and 5, respectively, is associated with decays from $\mathrm{Kr}^{+} 3 d^{9}{ }^{2} D_{3 / 2}$ to the $4 s^{2} 4 p^{3}{ }^{4} S$ ground state of $\mathrm{Kr}^{3+}$. At label 1 in Figs. 3 and 4 a sequence of peaks is visible between $\varepsilon_{3}=0$ and $2.0 \mathrm{eV}$ with the highest intensity at $\varepsilon_{3}=1.8 \mathrm{eV}$. The next band in Fig. 3 and the second spectrum from the top in Figs. 4 and 5 are associated with decays from $3 d^{9}{ }^{2} D_{5 / 2}$ and show a very similar structure. The main difference in the peak structure of the latter band is that here the intensity is strongest at $\varepsilon_{3}=2.0 \mathrm{eV}$. Since the electron kinetic energies from the Auger decays of the two initial core hole states are the same in the low-energy part as shown in Fig. 4, but differ by $1.27 \mathrm{eV}$ in the high-energy part as can be seen in Fig. 5, we conclude that the structures are connected to intermediate states in $\mathrm{Kr}^{2+}$ that lie close to $\mathrm{Kr}^{3+}$ $4 s^{2} 4 p^{3}{ }^{4} S$. We tentatively assign the sequence to Rydberg states (label $a$ in Fig. 2) converging onto the $4 s^{2} 4 p^{3}{ }^{2} D_{3 / 2}$ and ${ }^{2} D_{5 / 2}$ thresholds, which are located 2.11 and $2.32 \mathrm{eV}$ above the ${ }^{4} S$ ground state [25], respectively.

Two sharp intense peaks labeled 3 and 4 are observed at $\varepsilon_{3}=3.65 \mathrm{eV}$ with accompanying much weaker peaks at $\varepsilon_{3} \approx$ $3.96 \mathrm{eV}$. In Fig. 4, these peaks line up with the peaks labeled $5^{\prime}$ and $6^{\prime}$ in the two middle spectra, and the peaks $5^{\prime}$ and $6^{\prime}$ are also accompanied by similar weak peaks at higher $\varepsilon_{3}$. In Fig. 3 we observe that the spots $5^{\prime}$ and $6^{\prime}$ lie in the upper part of the bands and so are attributed to decays to ${ }^{2} D_{3 / 2}$ even though the $J=3 / 2$ and $J=5 / 2$ components cannot be resolved in the $y$ projection. The two spots are observed at $\varepsilon_{3}=1.55 \mathrm{eV}$ and since the ${ }^{2} D_{3 / 2}$ final state lies $2.11 \mathrm{eV}$ [25] above ${ }^{4} S$, we conclude that the spots $3,4,5^{\prime}$, and $6^{\prime}$ should be attributed to electrons originating from the Auger decay of a $\mathrm{Kr}^{2+}$ intermediate state $3.65 \mathrm{eV}$ above $4 s^{2} 4 p^{3}{ }^{4} S$, which we label $b$ in Fig. 2. The appearance of the weaker peaks at higher $\varepsilon_{3}$ could possibly reflect spin-orbit splitting of the intermediate state.

The labels 7 and 8 in Fig. 3 designate two intense spots in the decay to ${ }^{2} P_{3 / 2}$ at $\varepsilon_{3}=0.65 \mathrm{eV}$. Two accompanying spots located at $\varepsilon_{3}=0.95 \mathrm{eV}$, labeled $7^{\prime}$ and $8^{\prime}$, reflect decay to ${ }^{2} P_{1 / 2}$. A similar behavior is observed in the decays to ${ }^{2} D$ (middle part of the figure), where the strong spots in the decays to ${ }^{2} D_{3 / 2}$ labeled $5^{\prime}$ and $6^{\prime}$ at $\varepsilon_{3}=1.55 \mathrm{eV}$ have twin spots labeled 5 and 6 located at $\varepsilon_{3}=1.30 \mathrm{eV}$ in the decay to the ${ }^{2} D_{5 / 2}$ final state (lower part of the corresponding bands in the coincidence map). As can be seen in Fig. 3, the spots associated with the ${ }^{2} D_{3 / 2}$ final state are much more intense than the ones associated with ${ }^{2} D_{5 / 2}$. A particularly high intensity is thus acquired by the $J=3 / 2$ component for both ${ }^{2} P$ and ${ }^{2} D$ final states, which may suggest that angular momentum transfer is favorable in this case.

Two further observations can be made by inspection of the corresponding peaks in Fig. 4. Firstly, the strong peaks 7 and 8 do not line up with the strong peaks $3,4,5^{\prime}$, and $6^{\prime}$ in the upper part of the figure, and secondly, the latter peaks are assigned to an intermediate state, $b$, that has a binding energy relative to ${ }^{4} S$ that is lower than the binding energy of ${ }^{2} P$. Therefore, we associate the spots labeled $7,7^{\prime}, 8$, and $8^{\prime}$ with another intermediate state of $\mathrm{Kr}^{2+}$ with a binding energy of $0.65 \mathrm{eV}$ 
TABLE II. Auger cascades from $\mathrm{Kr}^{+} 3 d^{9}$ to $\mathrm{Kr}^{3+} 4 s^{2} 4 p^{3}$ observed in this study. The letters in the first column refer to the $\mathrm{Kr}^{2+}$ intermediate states deduced in this work and schematically drawn in Fig. 2, and the numbers in the second column refer to the feature labeling used in Figs. 3-5. The third and fourth column give the initial cationic and final tricationic state term symbols, and the kinetic energies of the two electrons measured in each double Auger decay cascade are given in columns five and six. The stated kinetic energies are given with an uncertainty of $50 \mathrm{meV}$ for energies below $4 \mathrm{eV}$ and $100 \mathrm{meV}$ for kinetic energies above $4 \mathrm{eV}$. In columns seven, eight, and nine, the energy of the intermediate state in each cascade is given relative to the $4 s^{2} 4 p^{3}{ }^{4} S \mathrm{Kr}^{3+}$ state, the initial single hole state, and the final tricationic state, respectively.

\begin{tabular}{|c|c|c|c|c|c|c|c|c|c|}
\hline \multicolumn{2}{|c|}{ Labels } & \multicolumn{2}{|c|}{ Electronic states } & \multicolumn{2}{|c|}{ Auger energy } & \multicolumn{4}{|c|}{ Energy level in $\mathrm{Kr}^{2+}(\mathrm{eV})$} \\
\hline Fig. 2 & Fig. 3 & $\begin{array}{c}\text { Initial } \\
3 d^{9} \mathrm{Kr}^{+}\end{array}$ & $\begin{array}{c}\text { Final } \\
4 s^{2} 4 p^{3} \mathrm{Kr}^{3+}\end{array}$ & $\varepsilon_{2}(\mathrm{eV})$ & $\varepsilon_{3}(\mathrm{eV})$ & $\begin{array}{c}\text { Above } \\
4 s^{2} 4 p^{3}{ }^{4} S_{3 / 2}\end{array}$ & $\begin{array}{c}\text { Below } \\
3 d^{9}{ }^{2} D_{5 / 2}\end{array}$ & $\begin{array}{c}\text { Below } \\
3 d^{9}{ }^{2} D_{3 / 2}\end{array}$ & $\begin{array}{c}\text { Above } \mathrm{Kr}^{3+} \\
\text { final state }\end{array}$ \\
\hline$a$ & 1 & $D_{3 / 2}$ & ${ }^{4} S_{3 / 2}$ & 18.9 & 1.75 & 1.75 & & 18.9 & 1.75 \\
\hline$a$ & 2 & $D_{5 / 2}$ & ${ }^{4} S_{3 / 2}$ & 17.4 & 2.00 & 2.00 & 17.4 & & 2.00 \\
\hline$b$ & 3 & $D_{3 / 2}$ & ${ }^{4} S_{3 / 2}$ & 17.0 & 3.65 & 3.65 & & 17.0 & 3.65 \\
\hline$b$ & 4 & $D_{5 / 2}$ & ${ }^{4} S_{3 / 2}$ & 15.8 & 3.65 & 3.65 & 15.8 & & 3.65 \\
\hline$b$ & 5 & $D_{3 / 2}$ & ${ }^{2} D_{5 / 2}$ & $\sim 17.0$ & 1.30 & 3.60 & & 17.0 & 1.30 \\
\hline$b$ & $5^{\prime}$ & $D_{3 / 2}$ & ${ }^{2} D_{3 / 2}$ & 17.0 & 1.55 & 3.65 & & 17.0 & 1.55 \\
\hline$b$ & 6 & $D_{5 / 2}$ & ${ }^{2} D_{5 / 2}$ & $\sim 15.7$ & 1.30 & 3.60 & 15.7 & & 1.30 \\
\hline$b$ & $6^{\prime}$ & $D_{5 / 2}$ & ${ }^{2} D_{3 / 2}$ & 15.7 & 1.55 & 3.65 & 15.7 & & 1.55 \\
\hline$c$ & 7 & $D_{3 / 2}$ & ${ }^{2} P_{3 / 2}$ & 15.9 & 0.65 & 4.80 & & 15.9 & 0.65 \\
\hline$c$ & $7^{\prime}$ & $D_{3 / 2}$ & ${ }^{2} P_{1 / 2}$ & 15.9 & 0.95 & 4.80 & & 15.9 & 0.95 \\
\hline$c$ & 8 & $D_{5 / 2}$ & ${ }^{2} P_{3 / 2}$ & 14.7 & 0.65 & 4.80 & 14.7 & & 0.65 \\
\hline$c$ & $8^{\prime}$ & $D_{5 / 2}$ & ${ }^{2} P_{1 / 2}$ & 14.7 & 0.95 & 4.80 & 14.7 & & 0.95 \\
\hline$c$ & 9 & $D_{3 / 2}$ & ${ }^{2} D$ & $\sim 15.6$ & $\sim 2.7$ & 4.9 & 15.6 & & 2.7 \\
\hline$c$ & 10 & $D_{3 / 2}$ & ${ }^{2} D$ & $\sim 14.3$ & $\sim 2.7$ & 4.9 & 14.3 & & 2.7 \\
\hline$g$ & 11 & $D_{5 / 2}$ & ${ }^{2} P_{3 / 2}$ & 10.4 & 5.0 & 14.5 & 5.0 & & 10.4 \\
\hline$g$ & 12 & $D_{3 / 2}$ & ${ }^{2} P_{3 / 2}$ & 10.4 & 6.2 & 14.5 & & 6.2 & 10.4 \\
\hline$g$ & 13 & $D_{5 / 2}$ & ${ }^{2} D_{5 / 2}$ & 12.2 & 5.0 & 14.5 & 5.0 & & 12.2 \\
\hline$g$ & 14 & $D_{3 / 2}$ & ${ }^{2} D_{5 / 2}$ & 12.2 & 6.2 & 14.5 & & 6.2 & 12.2 \\
\hline$c /(g)$ & 15 & $D_{5 / 2}$ & ${ }^{4} S_{3 / 2}$ & 14.6 & 4.8 & 4.8 & 14.6 & & 4.8 \\
\hline$e$ & 16 & $D_{5 / 2}$ & ${ }^{2} P_{3 / 2}$ & 7.85 & 7.50 & 12.0 & 7.50 & & 7.85 \\
\hline$e$ & 17 & $D_{3 / 2}$ & ${ }^{2} P_{3 / 2}$ & 8.7 & 7.85 & 12.0 & & 8.7 & 7.85 \\
\hline \multirow[t]{2}{*}{$f$} & 18 & $D_{5 / 2}$ & ${ }^{2} D_{5 / 2}$ & 10.6 & 6.50 & 12.9 & 6.50 & & 10.6 \\
\hline & $18^{\prime}$ & $D_{5 / 2}$ & ${ }^{2} D_{5 / 2}$ & 10.4 & 6.75 & & & & \\
\hline$e$ & 19 & $D_{5 / 2}$ & ${ }^{2} D_{5 / 2}$ & 9.5 & 7.55 & 11.8 & 7.55 & & 9.5 \\
\hline$d$ & 20 & $D_{5 / 2}$ & ${ }^{2} D_{5 / 2}$ & 8.6 & 8.45 & 10.9 & 8.6 & & 8.6 \\
\hline$f$ & 21 & $D_{3 / 2}$ & ${ }^{2} D_{5 / 2}$ & 10.6 & 7.75 & 12.9 & & 7.75 & 10.6 \\
\hline$d$ & 22 & $D_{3 / 2}$ & ${ }^{2} D_{5 / 2}$ & 9.7 & 8.60 & 10.9 & & 9.7 & 8.60 \\
\hline
\end{tabular}

relative to $4 s^{2} 4 p^{3}{ }^{2} P_{3 / 2}$. We label this state $c$ in Fig. 2 and in Table II.

The corresponding kinetic energies of the Auger electrons released in the decay from $\mathrm{Kr}^{+} 3 d^{9}$ to the intermediate state $c$, can be determined from Fig. 5 [labels (7) and (8)]. As expected, the kinetic energy $\varepsilon_{2}$ depends on the $J$ value of the initial $3 d$ hole state. For $3 d^{9}{ }^{2} D_{5 / 2}$ the kinetic energy is $\varepsilon_{2}=14.7 \mathrm{eV}$, which matches a line at $14.71 \mathrm{eV}$ found in the Auger spectrum by Aksela et al. [28]. They associated this finding with a transition from $3 d^{9}{ }^{2} D_{5 / 2}$ of $\mathrm{Kr}^{+}$to a ${ }^{1} S_{0}$ state with leading configuration $4 s^{0} 4 p^{6}$. According to our experimental data the $c$ state decays almost exclusively to the $4 s^{2} 4 p^{3}{ }^{2} P$ final state term, while the intensities in the decays to the ${ }^{4} S$ and ${ }^{2} D$ states, labeled 9,10 , and 15 in Fig. 3 are very low. This can be understood theoretically since a ${ }^{1} S \rightarrow$ ${ }^{4} S$ Auger decay is spin forbidden in the nonrelativistic limit. Moreover, for the decay into the ${ }^{2} D$ term Auger transitions are hampered since no partial wave with $J=1 / 2$ is allowed in this case. For the level $c$, both the NIST database [25] and the present multiconfiguration calculations disagree with the earlier assignment by Aksela et al. [28]. Our calculations suggest that level $c$ is a correlation satellite to the $4 s^{0} 4 p^{6}$ ${ }^{1} S_{0}$ state due to $4 p^{6} \leftrightarrow 4 s 4 p^{4} 4 d$ double excitations. For these satellites, the symmetry arguments regarding the intensity thus still apply. We now turn our attention to the comparatively weaker spots in the middle part of the coincidence map shown in Fig. 3. The spots 11, 13, and possibly 15 line up at $\varepsilon_{3}=5.0 \mathrm{eV}$, while the spots labeled 12 and 14 line up at $\varepsilon_{3}=6.2 \mathrm{eV}$. These spots remain at the same vertical position for different final states, but change position with the initial state. Furthermore, they do not move as a function of photon energy, as is found by our measurements at $h v=140 \mathrm{eV}$. We consequently attribute these features to a $\mathrm{Kr}^{2+}$ intermediate state, labeled $g$ in Fig. 2, with a binding energy approximately 5 eV below $3 d^{9}{ }^{2} D_{5 / 2}$.

On the right-hand side of the coincidence map shown in Fig. 3, the intensity is stronger again, particularly because of a number of distinct spots in the decays to ${ }^{2} D$ and ${ }^{2} P$. In this energy region it is more difficult to assign the observed spots to $\mathrm{Kr}^{2+}$ intermediate states since the kinetic energies released 


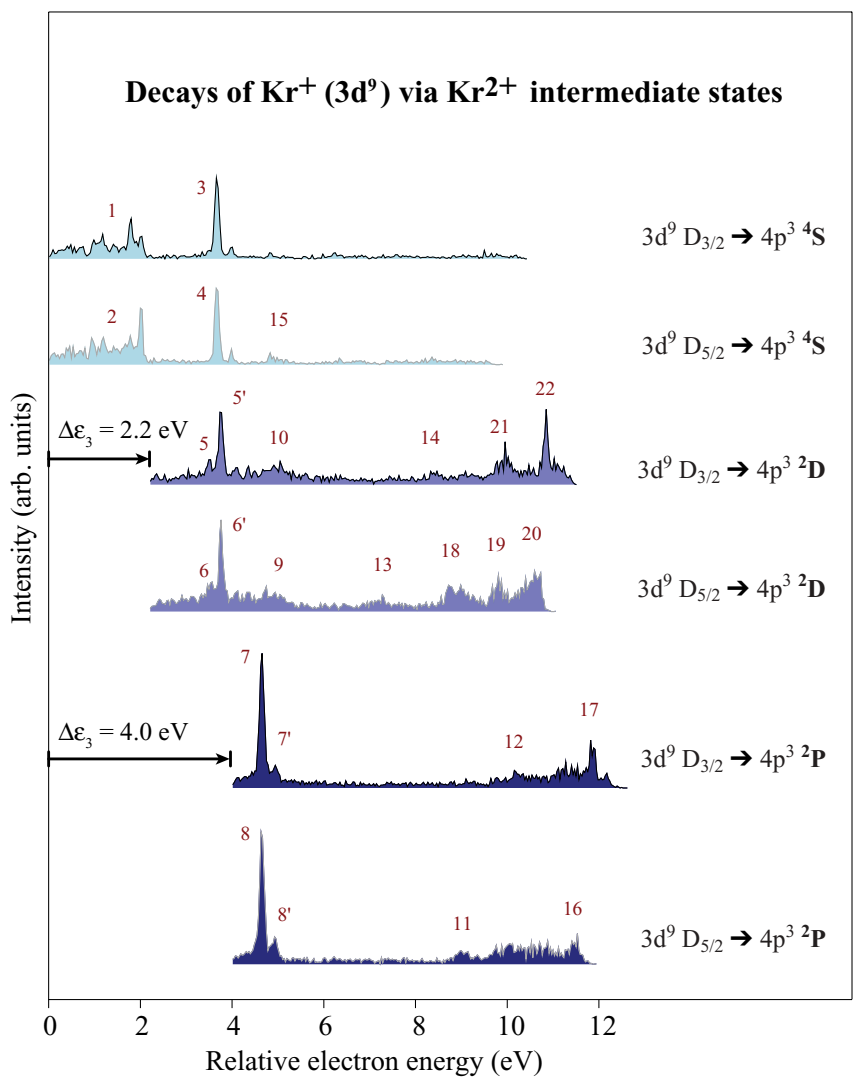

FIG. 4. (Color online) Spectra of the slower electron of two in the double Auger decays of $\mathrm{Kr}^{+} 3 d^{9}$ to $\mathrm{Kr}^{3+} 4 s^{2} 4 p^{3}$ plotted on a common intensity scale. The six spectra correspond to the six major bands in the coincidence map of Fig. 3. The kinetic energy of the electron has been shifted by the relative binding energy of the final state term referenced to the ${ }^{4} S$ tricationic ground state.

in each step are of the same size. (In the lower right corner of Fig. 3 there is no intensity because $\varepsilon_{3}$ is by definition smaller than $\varepsilon_{2}$ and this introduces a border at $\varepsilon_{3}=\varepsilon_{2}$ in the figure.) Labels 16 and 17 designate two spots very close to the border with $\varepsilon_{3}=7.50$ and $7.85 \mathrm{eV}$, respectively. The corresponding peaks in Fig. 4 do not line up, but the peak labeled (16) in Fig. 5 lies at the same kinetic energy $(7.85 \mathrm{eV})$ as the spot labeled 17 in Fig. 3. Addition of the kinetic energies $\varepsilon_{3}$, taken from Fig. 3, and $\varepsilon_{2}$, taken from Fig. 5, yields $7.50 \mathrm{eV}+7.85 \mathrm{eV}=$ $15.35 \mathrm{eV}$ for the features labeled 16 and $7.85 \mathrm{eV}+8.7 \mathrm{eV}=$ $16.55 \mathrm{eV}$ for the features labeled 17. This is in very good agreement with the tabulated spin-orbit splitting of $1.22 \mathrm{eV}$ for $\mathrm{Kr}^{+} 3 \mathrm{~d}^{9}$ [25]. We therefore conclude that there is a $\mathrm{Kr}^{2+}$ intermediate state located approximately $7.85 \mathrm{eV}$ above $\mathrm{Kr}^{3+}$ $4 s^{2} 4 p^{3}{ }^{2} P_{3 / 2}$.

Further up in the map there is a spot labeled 19 which lines up with the spot labeled 16 . The alignment is not perfect, but there is some uncertainty in the determination of the position of the spot labeled 16 since it lies close to the $\varepsilon_{3}=\varepsilon_{2}$ border. We tentatively associate the three spots 16,17 , and 19 with the same intermediate state, labeled $e$ in Table II and in Fig. 2. The spots 22 and 20 also seem to line up in the map. The latter is situated directly at the border, which suggests a common intermediate state (labelled $d$ in Table II and Fig. 2) exactly in the middle between $3 d^{9}{ }^{2} D_{5 / 2}$ and $4 s^{2} 4 p^{3}{ }^{2} D_{5 / 2}$. The

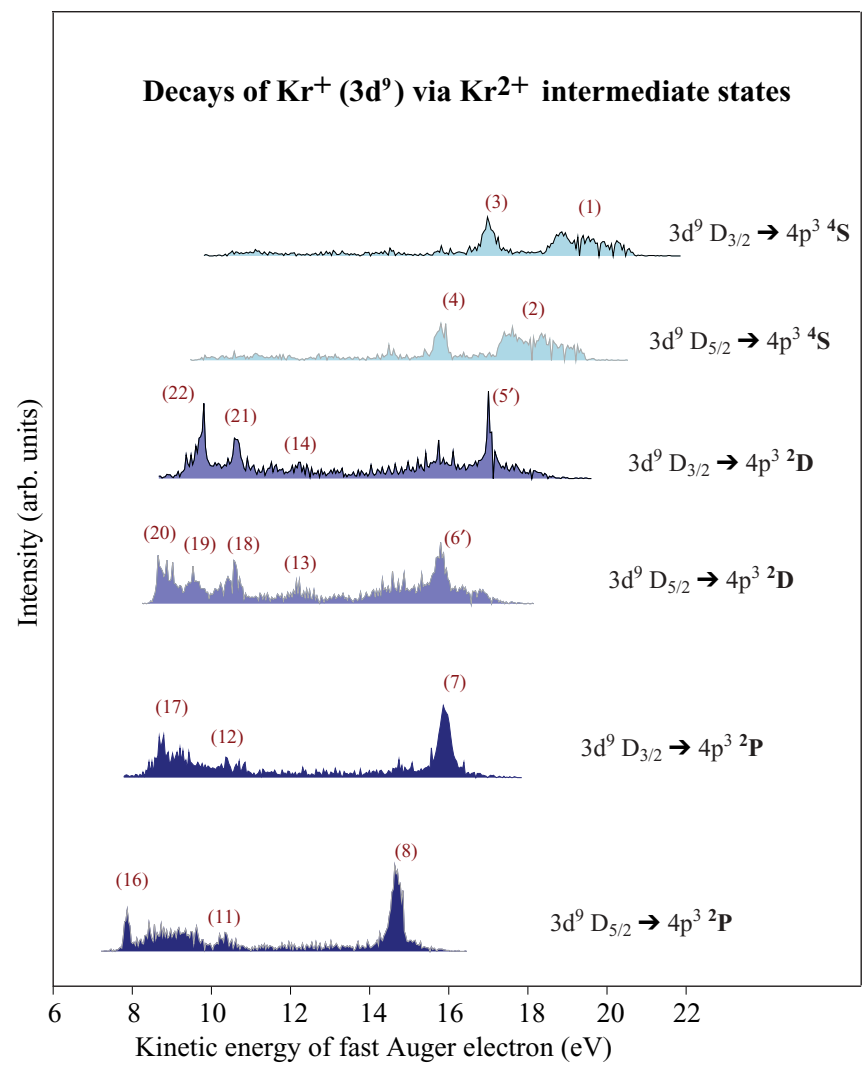

FIG. 5. (Color online) Spectra of the faster electron of two in the double Auger decay of $\mathrm{Kr}^{+} 3 d^{9}$ to $\mathrm{Kr}^{3+} 4 s^{2} 4 p^{3}$. These six spectra are the counterparts of the six spectra in Fig. 4. To facilitate comparisons, the same numbers (in parentheses) have been used as in Fig. 4.

energy of this state is $10.9 \mathrm{eV}$ relative to $4 s^{2} 4 p^{3}{ }^{4} \mathrm{~S}$. A similar observation was reported by Viefhaus et al. [9]. Label 18 marks a structured spot which is easier to characterise with the aid of Figs. 4 and 5. It is clear from Fig. 5 that it at least partially lines up with the structures labeled 21, suggesting an intermediate state, labeled $f$ in Table II and Fig. 2, with a binding energy of $10.6 \mathrm{eV}$ relative to $4 s^{2} 4 p^{3}{ }^{2} D_{5 / 2}\left(12.9 \mathrm{eV}\right.$ relative to $\left.{ }^{4} S\right)$.

In total we have tentatively identified seven intermediate states $a-g$ in $\mathrm{Kr}^{2+}$, where $g$ is particularly weak. They are summarized in Table III, and are schematically represented in Fig. 6 (which is an enlarged version of the right panel of Fig. 2) where also experimental branching ratios (peak heights) for the dominating transitions to the $4 p^{3} \mathrm{Kr}^{3+}$ states are given in parentheses (normalized to 10 for the strongest transition).

Figure 7 shows the observed intermediate states $a-g$ in comparison to $\mathrm{Kr}^{2+}$ energy levels computed as described in Sec. III. These levels have been calculated relative to the $\mathrm{Kr}^{2+}$ ground state, and are plotted in Fig. 7 relative to the $4 s^{2} 4 p^{3}$ ${ }^{4} S_{3 / 2}$ ground state of $\mathrm{Kr}^{3+}$, using the energy difference of $35.67 \mathrm{eV}$ between the $\mathrm{Kr}^{2+}$ [25] and $\mathrm{Kr}^{3+}$ [24] ground states. Theoretically, there are numerous $\mathrm{Kr}^{2+}$ intermediate energy levels, but the calculated Auger rates from the $\mathrm{Kr} 3 d^{9}$ initial hole state vary by several orders of magnitude, which suggests that only some of the intermediate levels are of primary importance. The rates of the second step of the Auger cascade also display pronounced variations. 
TABLE III. Observed intermediate $\mathrm{Kr}^{2+}$ states.

\begin{tabular}{|c|c|c|c|c|}
\hline \multirow{2}{*}{$\begin{array}{l}\text { Inter- } \\
\text { mediate }\end{array}$} & \multirow{2}{*}{$\begin{array}{l}\text { Observed energy } \\
\text { relative to } \\
\mathrm{Kr}^{3+}{ }^{4} S(\mathrm{eV})\end{array}$} & \multicolumn{2}{|c|}{$\begin{array}{c}\text { Final states of } \\
4 p^{-3} \mathrm{Kr}^{3+}\end{array}$} & \multirow[b]{2}{*}{ Comment } \\
\hline & & Dominant & Weak & \\
\hline$a$ & $0-2.0$ & ${ }^{4} S$ & & Rydberg states \\
\hline$b$ & 3.65 & ${ }^{2} D$ & ${ }^{4} S$ & \\
\hline$c$ & 4.80 & ${ }^{2} P$ & ${ }^{4} S,{ }^{2} D$ & \\
\hline$d$ & 10.9 & ${ }^{2} D$ & & \\
\hline$e$ & 12.0 & ${ }^{2} D,{ }^{2} P$ & & \\
\hline$f$ & 12.9 & ${ }^{2} D$ & $\left({ }^{2} P\right)$ & \\
\hline$g$ & 14.5 & & $\left({ }^{4} S\right),{ }^{2} D,{ }^{2} P$ & Low intensity \\
\hline
\end{tabular}

At calculated energies of 13.87 and $13.97 \mathrm{eV}$ above the $\mathrm{Kr}^{3+}$ ground state, there are two $\mathrm{Kr}^{2+}$ intermediate levels with high rates in both steps of the Auger cascade. According to the calculations of the present work, the state at $13.87 \mathrm{eV}$ has a major configuration $4 s 4 p^{4} 4 d{ }^{1} D_{2}$, while the state at $13.97 \mathrm{eV}$ is mainly $4 s 4 p^{4} 4 d{ }^{1} S_{0}$ but also has a contribution from the $4 p^{6}$ configuration with $30 \%$ weight. The states labeled $e$ and $f$ (cf. Table III) are observed at experimental energies of 12.0 and 12.9, respectively. Even though there is discrepancy between the calculated and the experimental energies, we tentatively ascribe the calculated states at 13.87 and $13.97 \mathrm{eV}$ to the states $e$ and $f$ since these have much higher intensity than state $g$. In addition, the experimental data reveal that the $e$ state decays to ${ }^{2} D_{5 / 2}$ and ${ }^{2} P_{3 / 2}$ and the $f$ state decays to ${ }^{2} D_{5 / 2}$ (cf. Table III), and neither to ${ }^{4} S_{3 / 2}$. The latter observation agrees well with the calculated results. A more detailed comparison shows marked deviations between the experimental and theoretical results; they may be due to the difficulties in constructing correct wave functions for the calculations. Further theoretical developments are needed to describe the hole-state resonances in the autoionization of atoms and ions in a (more) systematic manner. Beside additional and still missing bound-state correlations, such a description must include the coupling of the resonances to the continuum and a proper treatment of weak decay

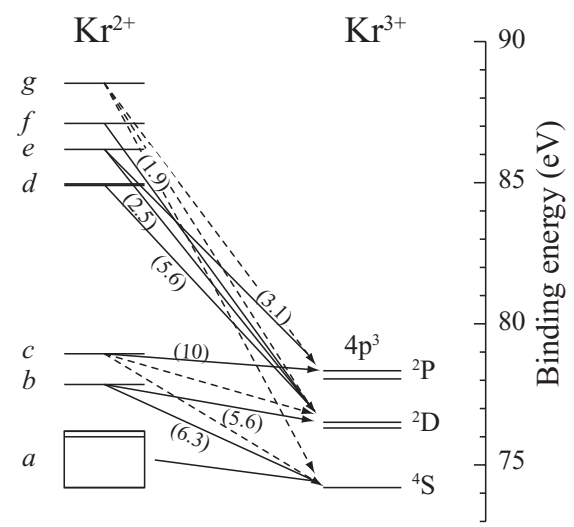

FIG. 6. Enlargement of the energy level diagram of Fig. 2, showing transitions from $\mathrm{Kr}^{2+}$ to $\mathrm{Kr}^{3+}$. Dominating transitions are indicated by solid arrows and weaker transitions by dashed arrows. Branching ratios (peak heights) for the dominating transitions are given in parentheses (normalized to 10 for the strongest transition).

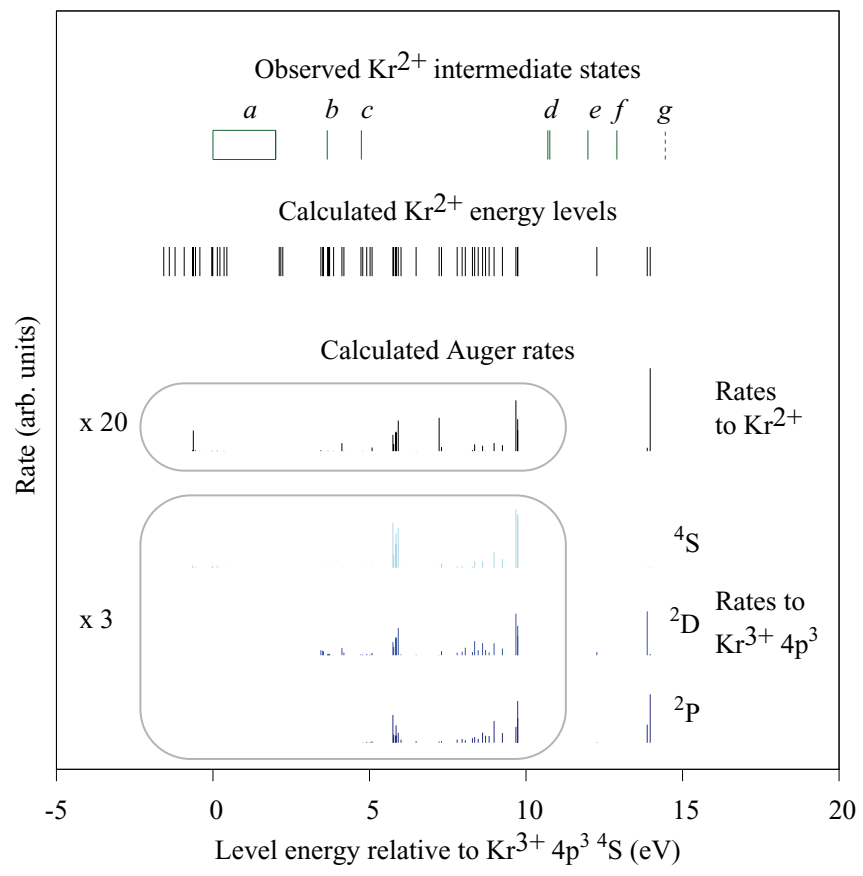

FIG. 7. (Color online) (Upper part) Experimentally observed and calculated $\mathrm{Kr}^{2+}$ intermediate states. (Lower part) Summed Auger rates calculated for transitions from $\mathrm{Kr}^{+} 3 d^{9}{ }^{2} D_{3 / 2,5 / 2}$ to the $\mathrm{Kr}^{2+}$ intermediate states, and the rates from these states to the $\mathrm{Kr}^{3+}$ final states with $4 s^{2} 4 p^{3}$ configuration are plotted using the same color coding as in Fig. 4. The transition rates to the two states at approximately $13.9 \mathrm{eV}$ are dominant. The rates to the states below $10 \mathrm{eV}$, which are very low, have been multiplied by a factor of 20 and 3 for the decays to and from $\mathrm{Kr}^{2+}$, respectively.

channels. Unfortunately, all the known techniques scale very unfavorably with the number of electrons, which makes them difficult to apply to real many-electron systems such as krypton. However, a comparison between the spectral features $b$ through $e, f$ with the Auger rates shows in both cases four groups of dominating lines. Based on this observation, the features $b, c$, and $d$ can tentatively be associated with the calculated lines at around 6.0, 7.0, and $9.5 \mathrm{eV}$, respectively. The experimental features labeled $a$ are attributed in the present interpretation to Rydberg states that were not considered in the calculations. All the remaining lines with lower Auger rates probably correspond to spectral features of somewhat lower intensity. The lines calculated between 2.5 and $5 \mathrm{eV}$ may thus be related to the spectral features 9 and 10 in Fig. 4 . Analogously, the lines predicted between 7.5 and $9 \mathrm{eV}$ can be associated with the intensity between the features 11 and 16 in Fig. 4.

We turn our attention once more to Fig. 1. As already mentioned previously, the features belonging to group III, with $\varepsilon_{2}+\varepsilon_{3}$ between 35 and $45 \mathrm{eV}$, present a quite complex structure. In order to disentangle the spectra, a second data set was measured at the photon energy of $140 \mathrm{eV}$, which we present in Figs. 8 and 9.

Figure 8 shows the spectrum of the second arrival electron corresponding to the coincidence events of group III in Fig. 1. The spectrum is plotted on a binding energy scale relative to the $3 d^{92} D_{5 / 2}$ level of $\mathrm{Kr}^{+}$in order to facilitate a comparison 


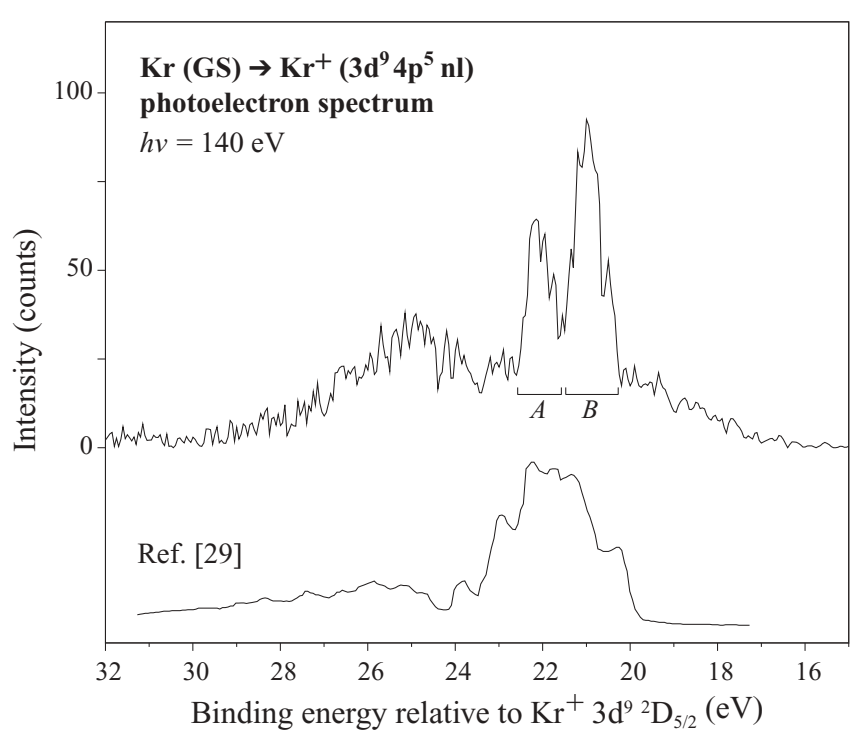

FIG. 8. Kr $3 d$ shake-up photoelectron spectrum measured at $h v=140 \mathrm{eV}$.

to the $\mathrm{Al} \mathrm{K} \alpha$-excited $\mathrm{Kr} 3 d^{9}$ shake-up photoelectron spectrum of Eriksson et al. [29] (also discussed in Ref. [30]), which is plotted in the lower part of this figure. Generally, the spectra are similar to each other, but there are differences in the relative intensities. These differences probably reflect the fact that not all Auger transitions are allowed to contribute intensity to the present spectrum. It is only those that decay to $\mathrm{Kr}^{3+} 4 s^{2} 4 p^{3}$ that are represented, whereas in the photoelectron spectrum of Eriksson et al. [29,30] all transitions are included irrespective of secondary processes in the system. Furthermore, the present spectrum has been measured at a photon energy much closer to the $\mathrm{Kr} 3 d$ threshold compared to the photon energy used by Eriksson et al. [29,30]. Hence, some of the differences may also be an effect of conjugate shake-up [29,30].

Figure 9 shows the spectra of the slowest electron of the coincidence events corresponding to group III in the same way that Fig. 4 shows the spectra of the slowest electron in group I. The spectra in Fig. 9 have been generated by selecting photoelectrons within the energy intervals of the two peaks in Fig. 8, marked $A$ and $B$, and the kinetic energy of the slowest electron, $\varepsilon_{3}$, has been shifted by the binding energy of the $4 s^{2} 4 p^{3}$ final state term relative to the ${ }^{4} S$ tricationic ground state. The two topmost spectra correspond to decays to ${ }^{4} S$, the middle ones correspond to decays to ${ }^{2} D$, and the two lowest spectra correspond to decays to ${ }^{2} P$ of the $4 s^{2} 4 p^{3}$ configuration. Overall, the signal is weaker than in Fig. 4, but some general remarks can still be made. The ${ }^{4} S$ final state seems to be populated almost entirely through the Rydberg-like intermediate states labeled $a$ in Fig. 2, which give rise to the intensity between 0 and $2 \mathrm{eV}$. In contrast, decays from the intermediate state $b$, which give rise to sharp peaks at $3.65 \mathrm{eV}$ in Fig. 4, are absent.

Decays to the ${ }^{2} D$ final state are more intense. In Fig. 4, sharp lines labeled $5^{\prime}$ and $6^{\prime}$ at $3.65 \mathrm{eV}$ are followed by a broad structure at higher energies, while in Fig. 9 the intensity at $3.65 \mathrm{eV}$ is rather low (akin to the decays to ${ }^{4} S$ ) and stronger at $4.3 \mathrm{eV}$. The spectra in Fig. 9 extend to higher energies than

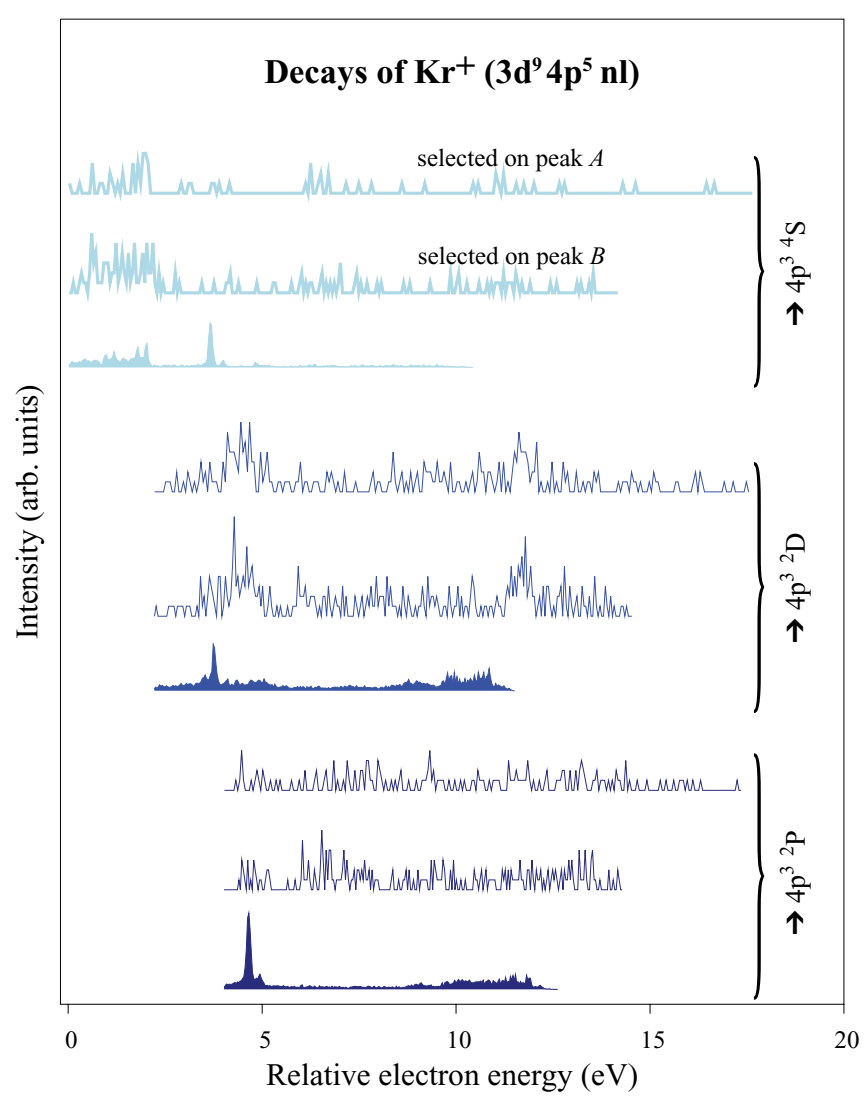

FIG. 9. (Color online) The slowest electron in the decay of $\mathrm{Kr}^{+} 3 d^{9} 4 p^{5} n l$ to $\mathrm{Kr}^{3+} 4 s^{2} 4 p^{3}$. All spectra have been measured in coincidence with photoelectrons from region $A$ or $B$ in Fig. 8. The kinetic energy of the electrons has been shifted by the relative binding energy of the final state term referenced to the ${ }^{4} S$ tricationic ground state. The filled curves show the spectra of the slowest electron in the decay of $\mathrm{Kr}^{+} 3 d^{9}$ (cf. top panel of Fig. 3).

in Fig. 4 because the $\varepsilon_{2}=\varepsilon_{3}$ border lies at higher energies. Hence, we can observe a peak at $11.6 \mathrm{eV}$ in the decays to ${ }^{2} D$ in Fig. 9 which lies beyond the border at the right-hand side of Fig. 4. We associate this peak with the $\mathrm{Kr}^{2+}$ intermediate state $e$, identified at label 19 in Fig. 4, where the slowest electron is emitted in the first step of the cascade Auger process, while in Fig. 9 it is emitted in the second step.

The decays to ${ }^{2} P$ in Fig. 9 are very weak with no apparent peaks. It is worth noting that the sharp peaks labeled 7 and 8 in Fig. 4 are absent in Fig. 9 in line with the absence of the peaks labeled $3,4,5^{\prime}$, and $6^{\prime}$ in the decays to ${ }^{4} S$ and ${ }^{2} D$.

\section{CONCLUSIONS}

We have used a magnetic bottle time-of-flight spectrometer to investigate the electron decay processes of $3 d$ ionized krypton leading to the $\mathrm{Kr}^{3+} 4 s^{2} 4 p^{3}$ final states. The decay is dominated by sequential (cascade) double Auger decays through discrete dicationic intermediate states. Coincidence measurements of the emitted electrons in combination with multiconfiguration Dirac-Fock calculations have enabled us to identify seven $\mathrm{Kr}^{2+}$ intermediate states and to determine their relative energies. 


\section{ACKNOWLEDGMENTS}

This work has been financially supported by the Swedish Research Council (VR), the Göran Gustafsson Foundation (UU/KTH), the Knut and Alice Wallenberg Foundation, and the Wenner-Gren Foundations, Sweden. J.H.D.E. thanks the Leverhulme Trust for financial support. S.F. acknowledges support from the FiDiPro program of the Finnish Academy. This work was also supported by the European CommunityResearch Infrastructure Action under the FP6 "Structuring the European Research Area" Programme (through the Integrated Infrastructure Initiative "Integrating Activity on Synchrotron and Free Electron Laser Science"-Contract No. R II 3-CT2004-506008).
[1] A. Einstein, Ann. Phys. 322, 132 (1905).

[2] P. Auger, J. Phys. Radium 6, 205 (1925).

[3] T. A. Carlson, Photoelectron and Auger Spectroscopy (Plenum, New York, 1975).

[4] T. A. Carlson and M. O. Krause, Phys. Rev. Lett. 14, 390 (1965).

[5] P. Kruit and F. H. Read, J. Phys. E: Sci. Instrum. 16, 313 (1983).

[6] J. H. D. Eland, O. Vieuxmaire, T. Kinugawa, P. Lablanquie, R. I. Hall, and F. Penent, Phys. Rev. Lett. 90, 053003 (2003).

[7] F. Penent, J. Palaudoux, P. Lablanquie, L. Andric, R. Feifel, and J. H. D. Eland, Phys. Rev. Lett. 95, 083002 (2005).

[8] P. Lablanquie, L. Andric, J. Palaudoux, U. Becker, M. Braune, J. Viefhaus, J. H. D. Eland, and F. Penent, J. Electron Spectrosc. Relat. Phenom. 156-158, 51 (2007).

[9] J. Viefhaus, M. Braune, S. Korica, A. Reinköster, D. Rolles, and U. Becker, J. Phys. B: At. Mol. Opt. Phys. 38, 3885 (2005).

[10] J. Palaudoux, P. Lablanquie, L. Andric, J. H. D. Eland, and F. Penent, J. Phys.: Conf. Ser. 141, 012012 (2008).

[11] K. J. S. Sawhney, F. Senf, M. Scheer, F. Schäfers, J. Bahrdt, A. Gaupp, and W. Gudat, Nucl. Instrum. Methods A 390, 395 (1997).

[12] E. Andersson, M. Stenrup, J. H. D. Eland, L. Hedin, M. Berglund, L. Karlsson, Å. Larson, H. Ågren, J.-E. Rubensson, and R. Feifel, Phys. Rev. A 78, 023409 (2008).

[13] [http://www.bessy.de].

[14] G. C. King, M. Tronc, F. H. Read, and R. C. Bradford, J. Phys. B: At. Mol. Opt. Phys. 10, 2479 (1977); 10, 3357 (1977).

[15] L. O. Werme, T. Bergmark, and K. Siegbahn, Phys. Scr. 6, 141 (1972).
[16] I. P. Grant, in Methods in Computational Chemistry, edited by S. Wilson (Plenum Press, New York, 1988), Vol. 2, p. 1.

[17] F. A. Parpia, C. Froese Fischer, and I. P. Grant, Comput. Phys. Commun. 94, 249 (1996).

[18] S. Fritzsche, C. Froese Fischer, and G. Gaigalas, Comput. Phys. Commun. 148, 103 (2002).

[19] M.-H. Chen, B. Crasemann, N. Mårtensson, and B. Johansson, Phys. Rev. A 31, 556 (1985).

[20] S. Fritzsche, J. Electron Spectrosc. Relat. Phenom. 114-116, 1155 (2001).

[21] G. Gaigalas, T. Zalandauskas, and S. Fritzsche, Comput. Phys. Commun. 157, 239 (2004).

[22] S. Fritzsche, B. Fricke, and W.-D. Sepp, Phys. Rev. A 45, 1465 (1992).

[23] S. Fritzsche, Phys. Scr., T 100, 37 (2002).

[24] J. H. D. Eland, P. Linusson, L. Hedin, E. Andersson, J.-E. Rubensson, and R. Feifel, Phys. Rev. A 78, 063423 (2008).

[25] NIST atomic spectra database [http://www.nist.gov/physlab/ data/asd.cfm].

[26] P. Bolognesi, L. Avaldi, M. C. A. Lopes, G. Dawber, G. C. King, M. A. MacDonald, C. Villani, and F. Tarantelli, Phys. Rev. A 64, 012701 (2001).

[27] E. Andersson et al. (in preparation).

[28] H. Aksela, S. Aksela, and H. Pulkkinen, Phys. Rev. A 30, 2456 (1984).

[29] B. Eriksson et al., J. Phys. Colloques 48, C9-531 (1987).

[30] S. Svensson, B. Eriksson, N. Mårtensson, G. Wendin, and U. Gelius, J. Electron Spectrosc. Relat. Phenom. 47, 327 (1988). 